\title{
LUT
}

University

\section{Synthesis of Micro-robotic Appendages Considering Different Performance Properties}

\author{
Milojević Andrija, Oldham Kenn
}

This is a Final draft version of a publication

published by Springer, Cham

in Microactuators, Microsensors and Micromechanisms. MAMM 2020

DOI: 10.1007/978-3-030-61652-6_12

Copyright of the original publication: () The Editor(s) and The Author(s) 2021

Please cite the publication as follows:

Milojević A., Oldham K. (2021) Synthesis of Micro-robotic Appendages Considering Different Performance Properties. In: Zentner L., Strehle S. (eds) Microactuators, Microsensors and Micromechanisms. MAMM 2020. Mechanisms and Machine Science, vol 96. Springer, Cham. https://doi.org/10.1007/978-3-030-61652-6_12

This is a parallel published version of an original publication. This version can differ from the original published article. 


\title{
Synthesis of Micro-Robotic Appendages Considering Different Performance Properties
}

\author{
Andrija Milojević ${ }^{1}$ and Kenn Oldham ${ }^{2}$ \\ ${ }^{1}$ LUT University, Lappeenranta 53850, Finland \\ ${ }^{2}$ University of Michigan, Ann Arbor MI 48109, USA \\ Andrija.Milojevic@lut.fi \\ oldhameumich.edu
}

\begin{abstract}
This paper presents a synthesis method for microelectromechanical system (MEMS) terrestrial micro-robotic appendages considering their different performance properties. In micro-robotics, actuation is often a challenge. Among candidate actuator types, thin-film lead-zirconate-titanite (PZT) may be adopted due to potential advantages in energy density and voltage and current requirements. Proposed micro-robotic appendages may be comprised of multiple micromachined materials: parylene-C (soft, passive), silicon (rigid, passive) a and thin-film PZT laminates (active). But a synthesis method for such type of micro-robots is lacking. In this paper, the topology optimization synthesis approach is introduced considering placement (type, dimensions, location, and orientation) of both active (thin-film PZT) and passive (parylene-C and $\mathrm{Si}$ ) multiple materials. The goal of the optimization is to realize micro-robotic appendages solutions, where transport ability and projected walking speed are maximized while needed electrical power is minimized. The individual steps in the synthesis process are presented together with different obtained solutions. Finite element method simulations of the micro-robotic appendage deformation behavior are presented as well.
\end{abstract}

Keywords: Micro-robot, Appendages, Synthesis, Thin-film PZT, MEMS.

\section{$1 \quad$ Introduction}

In many proposed robot applications, classical large-scale robots are not suitable and cannot be used. This is especially the case when robots need to access small and narrow places to perform functions such as inspection, delivery, sample collection, navigation of highly unstructured or hazardous areas, realization of targeted drug delivery, or exploration in lunar or space environments. For such kinds of applications, microrobots have been proposed [1-3]. The use of micro-robots offers many advantages including minimal disturbance to users, small power requirements, portability, disposability, and ability to work in large teams and exploit swarm intelligence. Thus, micro-robotics is a very relevant and ongoing research topic that has received much attention in recent years [1-8]. Among many different types of micro-robots [1, 2], MEMS-based terrestrial micro-robots are of special interest in this paper (Fig. 1a). 
Such kinds of robots are often produced with MEMS (microelectromechanical system) technologies where robot size usually ranges from few millimeters to 1-2 centimeters. MEMS micro-robots offer several advantages such as highly integrated compact systems and low-cost manufacturing due to batch fabrication.

But at the micro-scale, there is often a problem of providing actuation to the microrobots. Different types of actuation technologies have been proposed over time [4-6]. The realization of MEMS micro-robots or more specifically micro-robotic appendages with thin-film PZT as actuators (Fig. 1b) were of special interest in the authors' past work [7, 8]. Thin-film piezoelectric materials (PZT) offer many advantages including high actuation speeds, small power consumption, high work density per unit area, and reasonable efficiency as small capacitive loads [5], relative to other MEMS transducers. Drawbacks are limited payload, small inherent stroke lengths (a small range of motion for the micro-robot), and complex fabrication processes. It has been shown [7] that the use of thin-film PZT's in combination with other materials like polymer (soft) and silicon ( $\mathrm{Si}$, rigid) elements can overcome some of the disadvantages of PZT actuators. Moreover, it was shown that robustness and range-ofmotion of resulting micro-robotic appendage could be significantly improved.

Thus, this paper examines MEMS terrestrial micro-robots that realize their locomotion via appendages comprised of parylene-C (soft) and $\mathrm{Si}$ (rigid) structures as passive elements, and thin-film PZT as active elements (Fig. 1c and 1d). But this raises the question of how to design such micro-robotic appendages. Typically, microrobotic appendages are designed based on designer experience, through a trial-anderror approach or taking inspiration from nature (mostly insects) $[3,7,8]$. Realizing micro-robotic appendages in such a way often leads to non-optimized designs where the appendages' range of motion and performance are limited. Thus, the goal of this paper is to develop a synthesis method for the realization of optimized micro-robotic appendages. Here the designer would only need to specify desired initial parameters, and through the algorithmic procedure, the optimal solution would be automatically obtained, where prior design experience is not needed.

As a foundation for the synthesis method, the topology optimization approach is adopted here $[9,10]$. Topology optimization has been considered before for the design of MEMS-based structure as shown in many examples [9,11], but none has dealt with the synthesis of more complicated MEMS devices such as micro-robots. For the synthesis of micro-robotic appendages, placement (type, dimensions, location, orientation) of both active (thin-film PZT) and passive (parylene-C and Si) elements needs to be considered simultaneously in the optimization process. Moreover, here multiple materials need to be considered as well. In contrast, in [11] the actuators are added after the MEMS structure (comprised of passive elements) is obtained with optimization. Topology optimization including multiple materials was presented in [10], but not including the placement of active elements. In [12] the synthesis of microstructures comprised entirely of active elements is presented, but not including placement of passive multiple-material components. Further, synthesis of structures with simultaneous placement of both active and passive elements is considered in [13], but not including multiple materials or considering specific actuator type (like thin-film PZT). Continuum based topology optimization considering only multiple passive materials 
[11] and both active and passive materials (with limited placement variables) [14] has been realized before, but such methods are not applicable for the design of discrete like micro-robotic appendages. In general, the overall design methodology for the MEMS terrestrial micro-robots is lacking and many of the new design objectives need to be defined.

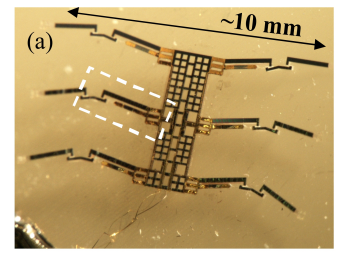

(c)

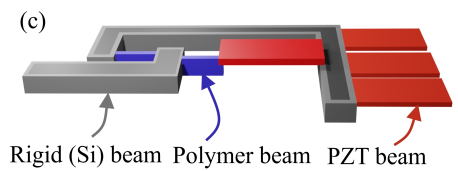

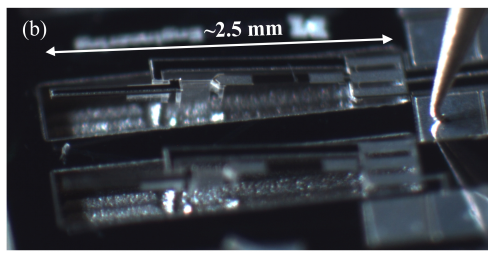

(d)

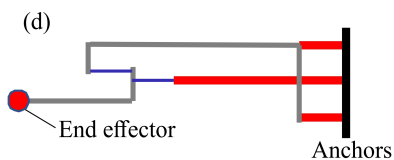

Fig. 1. a) MEMS terrestrial micro-robot prototype based on thin-film piezoelectric actuators with sample appendage design marked; b) micro-robot appendage tests structure composed of PZT; c) illustration of PZT, polymer and silicon structures arranged in sample appendage; d) illustration of appendage model, used in topology optimization framework [7].

In our previous paper [15] the basic topology optimization of the micro-robotic appendages that can realize control of the end-effector in different directions was presented. But only limited design possibilities were explored where the focus was on maximizing control of the appendages end-effector. In this paper a new synthesis method is presented for the realization of micro-robotic appendage designs, considering different performance characteristics like projected walking speed, transport ability, and total power (not done in [15]). The synthesis method will be presented, obtained solutions, together with Finite Element Method characterization of the selected micro-robotic appendage designs.

\section{Synthesis of MEMS terrestrial micro-robotic appendages}

The main goal of the synthesis is to realize optimal micro-robotic appendage solution with optimal performance properties. In other words, to obtained micro-robotic appendages designs that can realize high walking speeds, have good transport ability, and consume small power. From the synthesis perspective, micro-robotic appendages are comprised of (Fig. 1c and 1d):

(1) thin-film PZT as active elements, with three layers (top- Au layer, middlePZT layer, and bottom- $\mathrm{SiO} 2$ layer); when an appropriate voltage is applied the PZT contracts and bends,

(2) Si as rigid passive elements, formed where the silicon substrate is retained, 
(3) parylene-C as soft/elastic passive elements; these are formed from combined surface deposition and trench refill, forming beam-like elements with either T- or L- shaped cross-sections.

These elements correspond to the element types that have been co-fabricated with thin-film PZT in [7]. The aim of combining multiple material elements is: Si-rigid elements need to perform substantial weight-bearing, parylene-C soft/elastic element structure needs to amplify the small PZT input strokes, where both soft and rigid element structure should not expose the PZT to excessive forces from robot weightbearing.

The synthesis of the micro-robotic appendages includes finding the optimal distribution/arrangements (number, type, location, position, orientation) and dimensions (width, thickness, length) of the thin-film PZT, Si and parylene-C so that resulting appendage design can achieve the desired performances (high walking speed and transport ability, with minimal power requirements). To realize such synthesis the topology optimization approach is adopted $[10,13]$. Here the designer only needs to define the desired input parameters, then thought algorithmic procedures, the solution is automatically obtained.

The overview of the developed topology optimization of the micro-robotic appendages is illustrated in Fig. 2. The individual synthesis steps include:

(1) defining an initial problem - setting the desired input parameters (Fig. 2a),

(2) parameterization - translation of the defined problem into a set of variables that can be optimized (Fig. 2b and 2c),

(3) optimization process - applying a search algorithm to find the optimal solution of the defined problem (Fig. 2d, 2e, and 2f).

In the next subsections, these steps are described in detail.

\subsection{Problem definition}

As the first step in the synthesis process, the initial problem needs to be defined (Fig. 2a). This includes setting: the design domain (space in which the appendage solution needs to fit) where here the rectilinear shape is defined, location of the supports (the left end-edge of the designs domain is selected), location of the output (right-top-edge point is selected as end-effector of the appendage), inputs (input voltage is applied to activate the thin-film PZT), external loads that act on the micro-robotic appendage (here external loads are applied in both in-plane and out-of-plane direction), material properties for all the elements- thin-film PZT, parylene-C and Si (Young modulus), range of possible widths of all used active and passive elements, number of thin-film PZT and Si that will be used in the appendage structure, shape of parylene-C ( T or L shaped cross-section), together with other constraints and desired objects (like the desired volume of the end solution). The specific parameter values that are used in the synthesis of the micro-robotic appendages are given in Table 1.

\subsection{Parameterization}

The second step in the synthesis process is to realize the parameterization of the set problem. The design domain needs to be somehow represented with variables that can 
be optimized. Due to the discrete nature of the micro-robotic appendages design (Fig. 1d), here the ground structure approach (GSA) is adopted for the parameterization. Thus, the design domain is divided by nodes and spanned by beam-like elements connecting these nodes (Fig. 2b). This represents the initial solution within which optimal micro-robotic appendage performance is searched. Initially, all the elements represent passive parylene-C elements, where during optimization some of the elements are replaced with rigid Si elements and some with active thin-film PZT's (Fig. 2c). Elements are simultaneously turned on/off from the initial ground structure (Fig. 2d) until the optimal solution is yielded (Fig. 2e and 2f). Here multiple variables are defined: a variable that turns on/off paryelene-C elements, variable that defines a selection of active thin-film PZT, variable that defines a selection of passive rigid Si elements, and variable that defines the selection of parylene-C element type ( $\mathrm{T}$ or $\mathrm{L}$ shaped). The width of all the elements is optimized as well, thus one variable is defined for each of the elements that mark a possible range of widths. Additionally, the overall shape of the appendages is optimized by allowing nodes to wander (Fig. 2b) withing the predefined regions (the lengths and position of individual elements are changed). Thus, two variables are introduced that defines the possible range of node wandering in two directions $(x$ and $y)$ in in-plane (the plane of microstructure fabrication on a silicon wafer).

At the beginning, the initial ground structure represents a fully connected structure (Fig. 2b), where all the nodes are interconnected with one beam-like element. Such a ground structure usually leads to solutions that contain overlaying elements (one on top of the other, in two parallel planes). The micro-robotic appendage designs with such elements are difficult to fabricate in the MEMS domain. Thus, a filter is developed that searches and eliminates the overlaying elements from the structure, prior to the optimization. The reduced initial ground structure so obtained contains many overlapping intersecting elements (where elements cross each other while located in two parallel planes, one above the other). Such ground structure often can lead to appendage designs with intersecting elements, which is not desirable. Intersecting/crossing elements usually increase the overall stiffness of the end solution. This means that most of the thin-film PZT input actuation would be spent on deforming the structure thus only a small portion of the input displacement/forces would be transferred to the output. Moreover, appendage designs with intersecting elements are hard to fabricate. To avoid this, a simplified initial ground structure could be used, where one node is connected to only neighboring nodes (meaning that crossing elements do not exist or the number of such elements is small, where at the place of intersection nodes are defined). But such a simple initial net of elements cannot effectivity represent a design domain, and many of the potential good solutions are lost at the beginning. Another solution is to use the reduced initial ground structure but defining the nodes at the intersections, which is not desirable as solutions with small element length could be obtained (difficult to manufacture). Thus, to avoid the overlapping intersecting elements, a filter in the form of an objective goal is developed here. The filter searches and calculates the total number of intersections in the micro-robotic appendage system, where during the optimization this number is minimized.

The variables, initial net size, number of elements, and other parameters used in the synthesis of micro-robotic appendages are given in Table 1. 

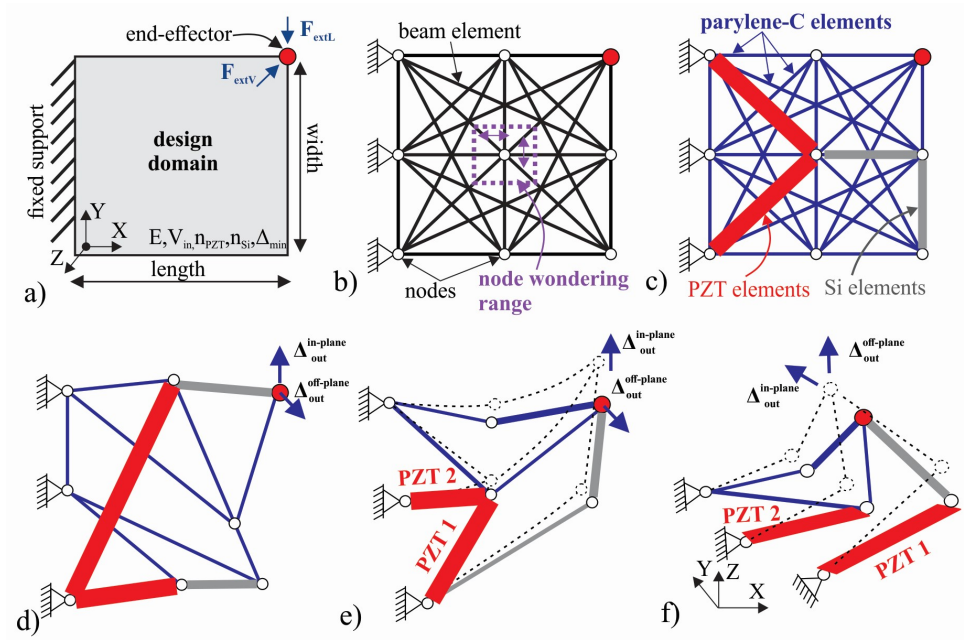

Fig. 2. Synthesis of the micro-robotic appendages: a) problem definition; b) parameterization of the design domain; c) initial ground structure with selected parylene-C, Si and thin-film PZT elements; d) optimization process (selection between different passive and active elements); e) example of the optimal solution shown in an initial (solid lines) and deformed (dash lines) state when all thin-film PZT's are active (planar view); f) optimal solution shown in space in an initial (solid) and deformed state (dashed), where end-effector realize displacement in both inplane and out-of-plane direction.

\subsection{Optimization and objective function}

As the last step in the synthesis of the micro-robotic appendages, a search algorithm needs to be applied to find the optimal solution of the given problem (Fig. 2e and 2f). Due to a large and complex design space that contains many variables, an optimization is applied as a search algorithm. Further, for the optimization, an objective function needs to be defined to guide the optimization algorithm towards the desired set goal.

The main aim of the optimization is to realize appendage designs that can achieve relatively high walking speeds, and good transport ability (payload capacity) of the resulting micro-robot while consuming low power. There are fundamental tradeoffs between these performance characteristics, as reducing power consumption reduces both speed and payload capacity, while higher payload capacities generally result in lower speeds. However, there are some opportunities to leverage the differences between internal piezoelectric deformation and point loads at the appendage tip, and dissimilar behavior in different axes to improve system-level performance. Thus, in the optimization, the walking speed and transport ability need to be maximized while the total required power need to be minimized. The resulting objective function for the synthesis of micro-robotic appendages is given in a form:

$$
\max \left[T_{\mathrm{ran}} \cdot w_{1}+V_{\mathrm{elW}} \cdot w_{2}-P_{\max } \cdot w_{3}-d_{\mathrm{ext}} \cdot w_{4}-n_{\mathrm{int}} \cdot w_{5}\right]
$$


The Eq. (1) is formulated so that transport ability $\left(T_{\text {ran }}\right)$ and projected walking speeds $\left(V_{\text {elw}}\right)$ are maximized, and total need power $\left(P_{\max }\right)$ is minimized. Further to increase the appendage payload capacity the end-effector displacement due to external loads $\left(d_{\text {ext }}\right)$ is minimized, and total number of intersections $\left(n_{\text {int }}\right)$ are minimized as well. The individual terms in the Eq. (1) are calculated by following set of equations:

$$
\begin{gathered}
T_{\mathrm{ran}}=\left(\frac{F_{\mathrm{extV}} \cdot d_{\mathrm{elV}}}{d_{\mathrm{FextV}} \cdot 9.8}-\frac{\sqrt{\frac{F_{\mathrm{exL}} \cdot d_{\mathrm{FextV}} \cdot 9.8}{d_{\mathrm{FextL}} \cdot F_{\mathrm{extV}} \cdot d_{\mathrm{elV}}}} \cdot \pi \cdot C_{\mathrm{ap}} \cdot V_{\mathrm{pzt}}^{2}}{2 \cdot B_{\mathrm{pw}}}\right) \cdot V_{\mathrm{elW}} \\
V_{\mathrm{elW}}=\sqrt{\frac{F_{\mathrm{exL}} \cdot d_{\mathrm{FextV}} \cdot 9.8}{d_{\mathrm{FextL}} \cdot F_{\mathrm{extV}} \cdot d_{\mathrm{elV}}}} \cdot \pi \cdot d_{\mathrm{elL}} / 2 \\
P_{\max }=n_{\mathrm{leg}} \cdot \sqrt{\frac{F_{\mathrm{exL}} \cdot d_{\mathrm{FextV}} \cdot 9.8}{d_{\mathrm{FextL}} \cdot F_{\mathrm{extV}} \cdot d_{\mathrm{elV}}}} \cdot \pi \cdot C_{\mathrm{ap}} \cdot V_{\mathrm{pzt}}^{2} / 2
\end{gathered}
$$

In brief Eq. (2) is based on the maximum deflection permitted due to gravity relative to appendage vertical range of motion, Eq. (3) is based on estimated natural frequency and appendage lateral range of motion, and Eq. (4) is based on estimated natural frequency and total PZT capacitance, where:

$d_{\mathrm{elV}}$ - end-effector displacement in the vertical direction (out-of-plane),

$d_{\mathrm{elL}}-$ end-effector displacement in the lateral direction (in-plane),

$d_{\text {FextV }}$ - end-effector displacement in vertical direction (out-of-plane) due to external loads,

$d_{\mathrm{Fext}}$ - end-effector displacement in the lateral direction (in-plane) due to external loads,

$V_{\mathrm{pzt}}$ - input voltage for the thin-film PZT's (Table 1),

$B_{\mathrm{pw}}$ - assumed battery power density (Table 1 ),

$C_{\text {ap }}$ - capacitance (calculated by the standard equation that can be found in [16]),

$n_{\text {leg }}-$ number of appendages.

All the terms in the objective function are calculated by using linear analysis which is implemented in the optimization algorithm. It is important to mention that this synthesis of micro-robotic appendages represents a multiobjective optimization problem, which is reduced to a single-objective problem by using weighting constants $\left(w_{\mathrm{i}}\right)$.

Among many different possible optimization methods, here a Genetic Algorithms (GA) are adopted $[9,13]$. GA's are very efficient in finding a global optimum over a large design space that contains many variables. Further, GA's represent a discrete optimization method, thus they are very suitable for optimization of discrete-like appendage designs where the variables are discrete as well. Moreover, a GA's are extensively used in the topology optimization problems $[9,13]$. The genetic parameters that are used for the synthesis of the micro-robotic appendages are an initial population of 200 , a total of 1000 generations, selection function type roulette, crossover probability of $95 \%$, elite count of 2 members, and mutation probability of $9 \%$. 
Table 1. Parameters used for the synthesis of the micro-robotic appendages.

\begin{tabular}{|c|c|}
\hline Parameter & case 2 \\
\hline design domain & $2000 \mu \mathrm{m} \times 2000 \mu \mathrm{m}$ \\
\hline grid size & $3 \times 3$ \\
\hline number of parylene-C el. & 28 \\
\hline $\begin{array}{l}\text { number of thin-film PZT and } \\
\text { Si el. }\end{array}$ & 2 \\
\hline input voltage for thin-film PZT & $15 \mathrm{~V}$ \\
\hline element modulus & $\begin{array}{c}\text { parylene-C, } E_{\text {pary }}=1.3 \mathrm{GPa} ; \\
\mathrm{Si}, E_{\mathrm{Si}}=170 \mathrm{GPa} ; \\
\mathrm{PZT}, E_{\text {base }}=80 \mathrm{GPa}, E_{\mathrm{PZT}}=56 \mathrm{GPa}, E_{\text {top }}=60 \mathrm{GPa}\end{array}$ \\
\hline element out-of-plane thickness & $\begin{array}{c}t_{\text {pary- }}=70 \mu \mathrm{m} ; t_{\text {pary }-\mathrm{L}}=5 \mu \mathrm{m} \\
t_{\mathrm{Si}}=30 \mu \mathrm{m}\end{array}$ \\
\hline range of element widths & $\begin{array}{c}t_{\text {base }}=0.5 \mu \mathrm{m}, t_{\mathrm{PZT}}=1 \mu \mathrm{m} ; t_{\text {top }}=1 \mu \mathrm{m} \\
w_{\text {pary }-\mathrm{T}}=3,5,7,9 \mu \mathrm{m} ; w_{\text {pary }-\mathrm{L}}=20,60,100 \mu \mathrm{m} \\
w_{\mathrm{Si}}=10,40 \mu \mathrm{m} \\
w_{\mathrm{PZT}}=50,100,150 \mu \mathrm{m}\end{array}$ \\
\hline node wandering space & $100 \times 100 \mu \mathrm{m}$ \\
\hline external loads & $F_{\mathrm{y}}=1 \mu \mathrm{N}, F_{\mathrm{z}}=1 \mu \mathrm{N}$ \\
\hline thin-film PZT el. range & $\begin{array}{l}\mathrm{PZT} 1=1-28 \\
\mathrm{PZT} 2=1-28\end{array}$ \\
\hline Si el. range & $\begin{array}{l}\mathrm{Si} 1=1-28 \\
\mathrm{Si} 2=1-28\end{array}$ \\
\hline parylene-C el. type & $\begin{array}{l}\text { parylene- } \mathrm{T} \text { type }=1-28 \\
\text { parylene- } \mathrm{L} \text { type }=1-28\end{array}$ \\
\hline assumed battery power density & $B_{\mathrm{pw}}=100(\mathrm{~W} / \mathrm{kg})$ \\
\hline weighting constants & $\begin{array}{c}w_{1}=1 \mathrm{e} 9-5 \mathrm{e} 9, w_{2}=1 \mathrm{e} 3-2 \mathrm{e} 3, w_{3}=0.3 \mathrm{e} 5-4 \mathrm{e} 5 \\
w_{4}=0.8, w_{5}=1 \mathrm{e} 3\end{array}$ \\
\hline
\end{tabular}

\subsection{Solutions of micro-robotic appendages}

Multiple GA's optimizations are run for a different set of initial parameters and values of weighting constants (Table 1) to obtain the micro-robotic appendage solutions. The optimization process starts with initial ground structure (Fig. 2b), where simultaneously some parylene-C elements are turned on/off from the structure, some are chosen as active thin-film PZT and some as passive rigid- Si elements, the width of individual elements are selected together with node locations/positions (Fig. $2 \mathrm{c}$ and $2 \mathrm{~d}$ ). The remaining parylene-C, thin-film PZT, and Si elements with corresponding dimensions and node locations form the optimal topology of the micro-robotic appendages (Fig. $2 \mathrm{e}$ and $2 \mathrm{f}$ ).

Fig. 3 shows some of the obtained solutions of the micro-robotic appendages. In all cases, the appendages realize appropriate output displacement of the end-effector, when all thin-film PZT's are activated at once. The performance characteristic for 
each obtained solution is depicted in Fig. 4. The appendage designs are compared against realized walking speed and required powered when end-effector displacement is considered. The results show that optimal solutions are very sensitive to weighting of the various performance metrics, but we see a tendency for locally optimal solutions to fall into three major categories: high stiffness, low displacement solutions with high transport capacity and high natural frequency, keeping speed high but with typically small step lengths and high power consumption (i.e. appendage 1 in Fig. 3, and solutions elevated on the z-axis of Fig. 4); low stiffness, high displacement solutions with modest projected walking speeds and large range-of-motion, but low transport capacity (i.e. appendages 2, 4 in Fig. 3, clustered near lower right of Fig. 4); and low displacement solutions having reduced active element size and natural frequency, for extremely low-power but low-speed locomotion (i.e. appendage 3 in Fig 3, clustered near lower left of Fig. 4).
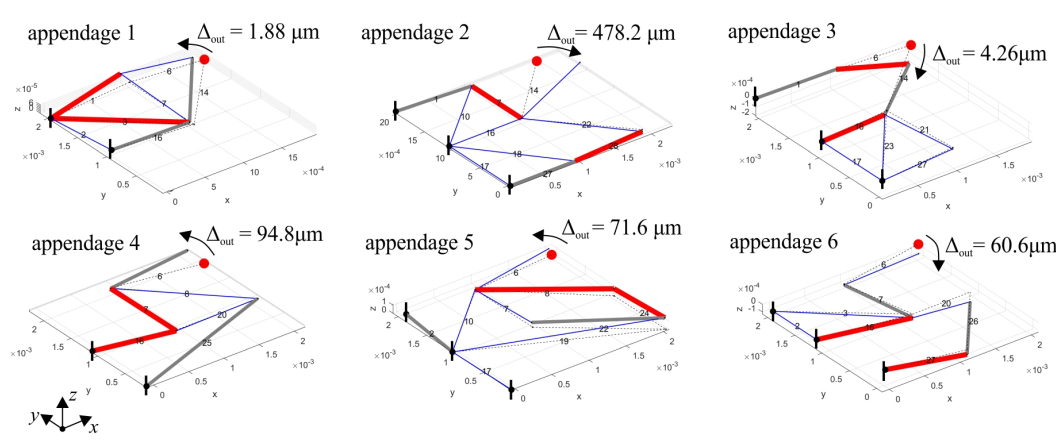

Fig. 3. Topology optimized solutions of the MEMS terrestrial micro-robotic appendages (a few representative obtained designs are shown).

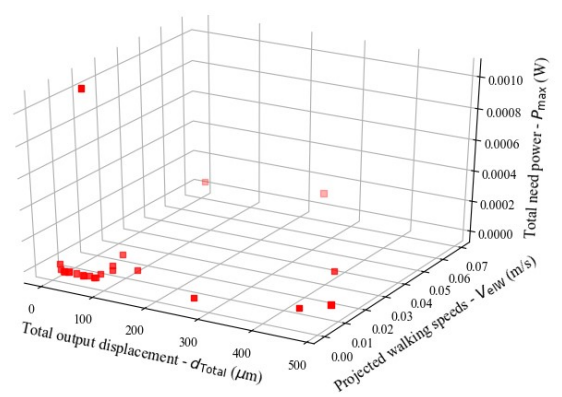

Fig. 4. The performance characteristics of the micro-robotic appendage solutions obtained with topology optimization.

\section{FEM based analysis of micro-robotic appendages}

To verify the micro-robotic appendage solutions (Fig. 3) Finite Element Method simulations are performed by using COMSOL Multiphysics. Based on the obtained solu- 
tions with optimization (Fig. 3), a 3D solid model of the appendages is designed considering the fabrication requirements. Here only a few of the selected designs are presented (Fig. 5). Like Fig. 1 and Fig. 3, micro-robotic appendages are comprised of:

- thin-film PZT active elements with three layers (top- Au, middle- PZT, bottom$\mathrm{SiO} 2$ ); marked in red (Fig. 5),

- Si passive rigid elements, enclosed in $\mathrm{SiO} 2$ trenches; marked in gray (Fig. 5),

- parylene-C passive soft elements; marked in blue (Fig. 5).
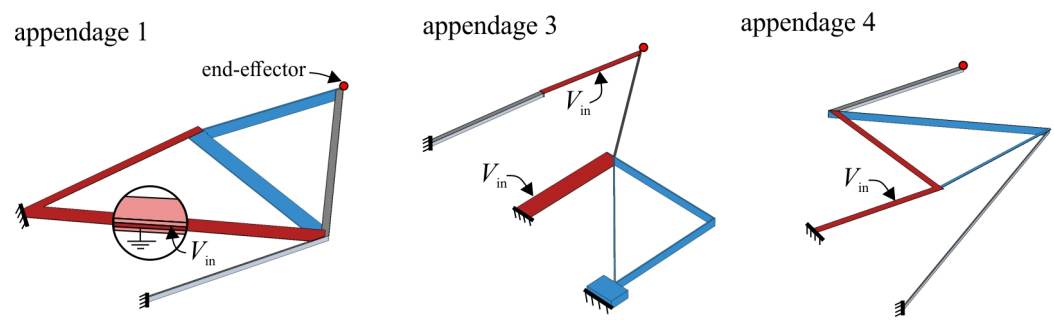

Fig. 5. A FEM setup and solid 3D model of the selected micro-robotic appendage designs, active thin-film PZT elements marked with red, passive (rigid) Si with gray, and passive (soft) parylene-C with blue color.

The deformation behavior together with the performance characteristic of the micro-robotic appendage designs is investigated. For the simulations in COMSOL, the stationary structural mechanics coupled with the piezoelectric module are used. The FEM setup is as follows: the fixed boundary condition (Fig. 5) is applied at the same location as in the corresponding topology optimized solution (Fig. 3); an input electric voltage of $15 \mathrm{~V}$ (the same value used in the optimization) is applied at the top surface of the middle-PZT layer, and ground (voltage equal to zero) is applied at the bottom surface of the middle-PZT layer (Fig. 5); the same material settings as in optimization are used for the corresponding type of elements. Additionally, the external loads are applied at the end-effector location, both in in-plane and out-of-plane direction. Two simulations are run for each of the investigated designs: for the case when all thinfilm PZT's are active, and for the case when only external loads are applied. The endeffector displacement is read out while the nonlinear analysis is considered. Fig. 6 shows the result of the deformation behavior of the micro-robotic appendages. It could be concluded that in all the simulated cases, the appendages realize appropriate output displacement of the end-effector when the input voltage is applied i.e. thin-film PZT's are activated. The results further show that similar deformation behavior is realized as in the case of topology optimization solutions (Fig. 3). The appendage performances are depicted in Fig. 7, where designs are compared against realized walking speed $\left(V_{\text {elW }}\right)$ and needed total power $\left(P_{\max }\right)$ considering total output displacement of the end-effector $\left(d_{\text {Total }}\right)$. The results in Fig. 7 , are calculated based on the read-out values of the end-effector displacement and using Eq. (2), (3), (4). The results further demonstrate representative designs of the types described in Section 2.4, with closest agreement between the low-power, low-speed appendage 3. The largerdisplacement designs, however, generate substantially less motion in Comsol simula- 
tion. We attribute this primarily to the difference between idealized connections between beam elements in topology and distributed intersections present in the solid model, with greater resistance to torsional motions and to lateral bending motions present between wider beams when fully modeled by FEA. Notably, projected walking speed does not decrease, because the reduced step length is offset by increased natural frequency (increased feasible step frequency) due to the increased stiffness in FEA results.

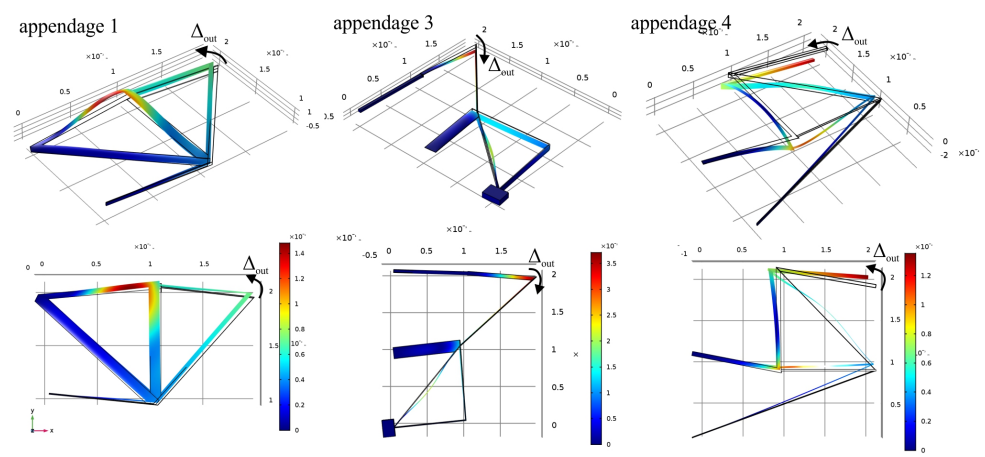

Fig. 6. FEM simulation results for the deformation behavior of the micro-robotic appendage solutions when the input voltage of $15 \mathrm{~V}$ is applied.

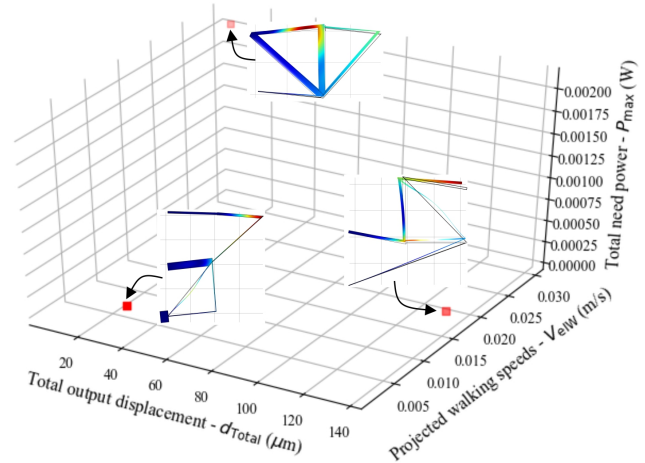

Fig. 7. FEM simulation results for performance characteristic of the selected micro-robotic appendage solutions.

\section{Conclusions}

This paper presents a novel synthesis method for the MEMS micro-robots and their corresponding appendages. It was shown that by using the developed optimization method a variety of solutions for appendage design could be realized in response to varying weighting of candidate performance specifications, such as transport ability, walking speed, range-of-motion, and/or power budget. Future work may analyze 
combined behavior through metrics such as cost-of-transport, detailed gait analysis and control in simulation of full multi-legged robots, and physical prototyping of select appendage and robot designs.

\section{References}

1. Palagi, S., Fischer, P.: Bioinspired microrobots. Nat Rev Mater 3, 113-124 (2018).

2. Sitti, M., Wiersma, D. S.: Pros and Cons: Magnetic versus Optical Microrobots. Adv. Mater. 2020, $1906766(2020)$

3. Oldham, K., Pulskamp, J., Polcawich, R., Ranade, P., Dubey, M.: Thin-film piezoelectric actuators for bio-inspired micro-robotic applications. Integrated Ferroelectrics 1(95), 54 65 (2007).

4. Huber, J. E., Fleck, N. A., Ashby, M. F.: The selection of mechanical actuators based on performance indices. In: Proc. Mathematical, Physical and Engineering Sciences 453(1965), pp. 2185-2205 (1997).

5. Edamana, B., Oldham, K. R.: Optimal low-power piezoelectric actuator control with charge recovery for a microrobotics leg. IEEE/ASME Transactions on Mechatronics 18 (1), 251-262 (2013).

6. Murthy, R., Das, A. N., Popa, D. O., Stephanou, H. E.: ARRIpede: An assembled die-scale microcrawler. Advanced Robotics 25, $965-990$ (2011).

7. Choi, J., Shin, M., Rudy, R. Q., Kao, C., Pulskamp, J. S., Polcawich, R. G., Oldham, K. R.: Thin-film piezoelectric and highaspect ratio polymer leg mechanisms for millimeter-scale robotics. International Journal of Intelligent Robotics and Applications 1 (2), 180-194 (2017).

8. Qu, J., Choi, J., Oldham, K. R.: Dynamics structural and contact modeling for a silicon hexapod microrobot. Journal of Mechanisms and Robotics 9(6), pp. 061006 (2017).

9. Howell, L. L., Magleby, S. P., Olsen, B. M.: Handbook of Compliant Mechanisms, Chichester: John Wiley \& Sons (2013).

10. Saxena, A.: Topology design of large displacement compliant mechanisms with multiple materials and multiple output ports. Structural and Multidisciplinary Optimization 30, 477-490 (2005).

11. Ruiz, D., Sigmund, O.: Optimal design of robust piezoelectric microgrippers undergoing large displacements. Structural and Multidisciplinary Optimization 57, 71-82 (2018).

12. Moussa, R. E. K., Grossard, M., Boukallel, M., Chaillet, N., Hubert, A.: Observationoriented design of a monolithic piezoelectric microactuator with optimally integrated sensor. In: 41st International Symposium on Robotics and ROTOTIK 2010, pp. 954-961. Verlag 1, Jun 2010, Munich, Germany (2010).

13. Milojević, A., Pavlović, N. D.: Development of a new adaptive shape morphing compliant structure with embedded actuators. Journal of Intelligent Material Systems and Structures 27, 1306-1328 (2016).

14. Wang, Y., Luo, Z., Zhang, X., Kang, Z.: Topological design of compliant smart structures with embedded movable actuators. Smart Materials and Structures 23(4), 045024 (15pp) (2014).

15. Milojević, A., Krokhmal, V., Wu, B., Oldham, K.: Topology Optimization of Microrobotic Appendages Combining Piezoelectric, Polymer, and Silicon Beams. In: 2019 International Conference on Manipulation, Automation and Robotics at Small Scales (MARSS), pp. 1-6. Helsinki, Finland (2019).

16. K. R. Oldham, J. S. Pulskamp, R. G. Polcawich, and M. Dubey, "Thinfilm PZT lateral actuators with extended stroke," Journal of Microelectromechanical Systems, vol. 17, no. 4, pp. 890-899, Aug 2008. 